\title{
Treatment Options for Advanced Colorectal Cancer Continue to Improve
}

Since its introduction more than 40 years ago, fluorouracil (FU) has been the mainstay of systemic therapy for colorectal cancer. After a long period of evaluation, it was confirmed that FU-based therapy prolonged survival and improved the quality of life of patients with metastatic disease compared to those receiving best supportive care alone. However, the overall therapeutic gain has remained meagre. Even if used in an optimal fashion, i.e., by continuous intravenous infusion with leucovorin (LV) modulation, fewer than a third of the patients achieve an objective response, and median survival is only about 12 months.

The position of FU/LV as standard first-line treatment has already been challenged some time ago by the development of several new agents that are non-crossresistant and act synergistically with FU, or have more favourable toxicity profiles or methods of administration. Among these new drugs are folatebased thymidilate synthase inhibitors such as raltitrexed, the oral fluorinated pyrimidines, the semi-synthetic topoisomerase I inhibitor irinotecan, and the third-generation platinum compound oxaliplatin. Encouraging phase II data of these new drugs and combinations in advanced colorectal cancer now have been confirmed in several large randomised European and US trials, mature results of which have been reported only this May, during the annual meeting of the American Society of Clinical Oncology in Atlanta. Significant new trial data, in fact, have been presented that are to change our thinking about the best current methods of treating advanced colorectal cancer.

In two multicenter phase III trials involving $>1,100$ patients, the oral fluoropyrimidine uracil-ftorafur (UFT, Orzel $\left.{ }^{\circledR}\right)+$ LV was compared with the bolus FU/LV Mayo Clinic regimen. In both trials, there was no difference in terms of objective response rate, time to progression, and overall survival. Patients receiving Orzel/LV, however, experienced significantly less febrile neutropenia and mucositis. For another FU prodrug, capecitabine $\left(\mathrm{Xeloda}^{\circledR}\right)$ that has already gained approval for another indication, refractory breast cancer, even more encouraging results have been reported. In two large parallel studies, each involving 600 previously untreated colorectal cancer patients, Xeloda was prospectively compared with bolus FU/LV. In both trials, not only a more favourable tox- icity profile with less severe adverse reactions was noted in favour of the prodrug, but Xeloda also resulted in significantly higher objective response rates ( 27 vs. $18 \%$ and 23 vs. $15 \%$ ). Time to progression and overall survival were similar. Even if not yet prospectively compared to continuous infusional FU regimens, these fluoropyrimidine prodrugs seem to hold the promise of equal efficacy, better tolerance, greater convenience, and reduced costs due to the oral mode of administration.

An update of a European phase III trial involving 420 previously untreated patients was provided that has demonstrated a significant benefit for adding oxaliplatin to infusional FU/LV (deGramont regimen): objective response rate was 51 vs. $22 \%$, and progression-free survival was 35 vs. 24 weeks $(\mathrm{p}=0.0001)$, clearly favouring the oxaliplatin-combination arm. Its superiority in terms of overall survival (17 months) became only evident by multivariate analysis, since several patients failing FU/LV were crossed over to oxaliplatin or other therapy. A doubling of the objective response rate and a clinically meaningful prolongation of progression-free and overall survival, finally have also been documented for the combination of the semi-synthetic camptothecin derivative irinotecan with FU/LV. In 3 independent phase III trials comparing bolus or infusional FU/LV (AIO or deGramont regimen) with and without irinotecan, significantly better therapeutic results have been obtained for the combination: objective response rates were 39,40 , and $49 \%$, and median survival times in the range of 14.4 to 17 months. The tolerance of treatment with these novel drug combinations was acceptable. Though certain severe adverse reactions were more commonly noted, serial assessment of quality of life revealed no difference compared to the FU/LV control arm.

Thanks to world-wide co-operative clinical investigational efforts, these important data have been created within a relatively short period of time, and they do suggest that it may has become time once more to reconsider/modify our current methods of treating patients with newly diagnosed advanced colorectal cancer. In patients with tumour-related symptoms, in those with rapidly progressive diseases with visceral or peritoneal metastases, as well as in the neoadjuvant setting, the new FU/LV combination regimens with oxaliplatin or irinotecan should be preferred; compared to the traditional standard,

\begin{tabular}{ll}
\hline KARGER & $\oplus 1999$ S. Karger GmbH, Freiburg \\
$\begin{array}{l}\text { Fax +49 7614520714 } \\
\begin{array}{l}\text { E-mail kargergmbh@aol.com } \\
\text { www.karger.com }\end{array}\end{array}$ & $\begin{array}{l}\text { Accessible online at: } \\
\text { http://BioMedNet.com/karger }\end{array}$
\end{tabular}


they duplicate our chance of obtaining an immediate palliative treatment success. In patients with less aggressive tumours, without clinical symptoms, and particularly if they are aged, raltitrexed and as soon as approved for this indication, the oral FU prodrugs are likely to become the new treatment of choice. Due to its proven superior response activity and tolerance, as well as ease of administration, Xeloda, in fact, will probably become the tamoxifen for colorectal cancer. In case of treatment failure, effective second-line therapy with oxaliplatin or irinotecan still can be offered at least to some of these patients.

Today it seems obvious that we have more and better treatment options for advanced colorectal cancer than ever before, but there are a number of unanswered questions and we still have to learn more about the new drugs' and combinations' optimal use for treating our patients. What is the therapeutic role of other new agents with novel mechanisms of action still under investigation, such as HMAF, LY 295501, bizelesin, ONYX-015, inhibitors of angiogenesis and signal transduction plus other gene therapy strategies? Which of the recently established novel chemotherapeutic regimens provides the best balance between clinical activity and toxicity, what is the optimal duration of palliative treatment, and are there other molecular markers in addition to intratumoural thymidilate synthase expression that can be used routinely to prospectively identify patients who are most likely to respond? In the near future, it will also be important to translate the available better treatment options into the postoperative adjuvant setting. With use of the new more active regimens, there is hope that the long-term prognosis of patients with lymph-node-positive colorectal cancer can be further improved, and that the benefit of adjuvant treatment also can be demonstrated conclusively in those with stage II disease. Clinical trials to answer these and other questions regarding the optimal management of patients with colorectal cancer are under way or are scheduled to begin soon. Undoubtedly, this is an exciting time for all those involved in treating colorectal cancer.

W. Scheithauer, Wien 